\title{
CATALISI IN SISTEMI MICELLARI AUTO-ASSEMBLATI
}

\author{
Nota del s.c. GIORGIO STRUKUL (*)
}

(Adunanza dell'11aprile 2013)

SunTO. - I sistemi micellari sono un nuovo, valido e versatile mezzo in cui condurre reazioni catalitiche organiche che consente di eliminare i solventi organici, di indurre una migliore attività e selettività nel catalizzatore, di migliorare le rese, ridurre gli scarti, semplificare il processo, risparmiare energia, operando in maniera più sostenibile dal punto di vista economico ed ambientale.

$* * *$

ABSTRACT. - Micellar systems are a new versatile medium to carry out catalytic organic reactions, that allows to eliminate organic solvents, induce a better catalyst activity and selectivity, improve yields, reduce waste, thereby simplifying processes, saving energy and operating in a more sustainable manner both from the economical and environmental viewpoint.

\section{IL RUOLO DEL SOLVENTE NELLA CHIMICA ORGANICA INDUSTRIALE}

Nel corso degli ultimi vent'anni la necessità di proteggere l'ambiente in cui viviamo ha posto i chimici di fronte ad una serie di sfide tese a ripensare le modalità di produzione dell'industria chimica, cer-

(*) Dipartimento di Scienze Molecolari e Nanosistemi, Università Ca' Foscari, 30123 Venezia, Italy.

E-mail: strukul@unive.it 
cando vie alternative meno inquinanti, l'uso di catalizzatori per semplificare le procedure, migliorando le rese, riducendo le emissioni e il consumo di energia. Nel valutare l'impatto ambientale di una reazione chimica si fa molto spesso ricorso a parametri quali l'economia atomica [1] o il fattore E.[2] Essi esprimono rispettivamente il rapporto fra il peso molecolare del prodotto desiderato relativamente alla somma dei pesi molecolari dei reagenti (economia atomica) e il rapporto fra la massa degli scarti prodotti da una reazione chimica e la massa del prodotto di interesse (fattore $\mathrm{E}$ ) assumendo che le reazioni vadano a completezza e in assenza di sottoprodotti indesiderati. Naturalmente maggiore sarà l'economia atomica (o minore il fattore E) maggiore sarà la sostenibilità (teorica) del processo considerato. In campo farmaceutico, dove la sintesi di principi attivi è molto spesso caratterizzata da una sequenza di reazioni chimiche, il fattore $\mathrm{E}$ può arrivare anche a 100, il che significa che in condizioni ideali e puramente teoriche $(100 \%$ di conversione in tutte le reazioni in sequenza) per ogni kg di prodotto utile si produce un quintale di scarti che vanno smaltiti, bruciati, etc.. In pratica le quantità di scarti prodotti sono molto maggiori.

La sintesi organica in settori quali quelli dei fine chemicals e dei farmaci è caratterizzata dall'uso massiccio di reazioni condotte in soluzione, spesso in presenza di catalizzatori solubili. Fra i constituenti di una reazione in fase liquida il solvente è quello presente in quantità maggiore (circa l' $85 \%$ della massa totale) e il suo recupero e riciclo a fine reazione ha un'efficienza che raramente supera 1'80\%. Quindi la valutazione della compatibilità ambientale del solvente è un elemento di informazione molto importante. Per illustrare questo punto un esempio calzante è costituito dall'industria chimica svizzera poichè essa è basata principalmente sulla produzione di fine chemicals e farmaci. Secondo un'analisi recente [3] il 28\% dei solventi impiegati viene riciclato, il $4 \%$ passa all'impianto di smaltimento dei reflui e il $68 \%$ viene combusto per recuperare energia. Quanto ai tipi di solventi impiegati toluene, acetone e metanolo costituiscono circa il $65 \%$ del totale. Non a caso, ovviamente, poichè sono fra quelli col più basso impatto sull'ambiente e sull'uomo e il bilancio più favorevole fra l'energia spesa per la loro produzione e quella ricavata dalla loro combustione o risparmiata tramite riciclo. Sotto questo profilo il solvente ideale sarebbe l'acqua. Essa presenta una serie di vantaggi difficilmente emulabili da altri tipi di solvente: (i) è sicura per l'ambiente, non è infiammable, non è tossica ed è a basso costo; (ii) è particolarmente adatta per reazioni in cui sia già presente nei reagenti (ad es. 
nelle ossidazioni con $\mathrm{H}_{2} \mathrm{O}_{2}$, nelle reazioni di idratazione, etc.); (iii) induce il cosiddetto effetto idrofobico che può consentire una maggiore attività e selettività nella reazione studiata; (iv) è polare e quindi facilita la separazione del catalizzatore sciolto in acqua quando si usino mezzi bifasici acqua/solvente organico. Purtroppo, a fronte di tutte queste interessanti proprietà, l'acqua ha scarse capacità solventi nei confronti della maggior parte dei substrati organici e dei catalizzatori e reagisce irreversibilmente con alcuni gruppi funzionali (specialmente organometallici). Questo spiega perchè nelle reazioni organiche il suo impiego come solvente sia molto limitato.

In letteratura sono state suggerite varie strategie tese a rendere un sistema catalitico compatibile con l'acqua,[4] le principali sono: (i) l'uso di solventi organici miscibili con acqua (ad es. etanolo, tetraidrofurano, dimetilsofossido), che ovviamente non elimina i problemi legati alla loro presenza, rende il loro recupero ancor più difficoltoso ed è di applicabilità piuttosto limitata; (ii) l'uso di catalizzatori intrinsecamente solubili in acqua (purtroppo applicabile solo in pochi casi); (iii) la modifica dei leganti presenti sul catalizzatore con gruppi fortemente idrofili tali da rendere il sistema idrosolubile. Quest'ultimo è stato l'approccio più usato ed è effettivamente applicabile ad una gamma piuttosto ampia di catalizzatori. Richiede però tempo e fatica per la sintesi dei nuovi leganti modificati e può alterarne le proprietà steriche ed elettroniche influenzando negativamente l'attività catalitica. Tuttavia è l'unico che ha finora condotto ad un'applicazione industriale nel processo Ruhrchemie-Rhone Poulenc per la sintesi della n-butirraldeide tramite idroformilazione impiegando come catalizzatori complessi di rodio idrosolubili. Il catalizzatore può essere separato e riciclato.[5]

\section{I SISTEMI MICELLARI}

In Natura tutte le reazioni enzimatiche avvengono in acqua. Gli enzimi sono delle macromolecole anfifiliche che contengono sia gruppi funzionali idrofili che porzioni fortemente idrofobe. La loro compatibilità con il mezzo acquoso in cui si trovano ad operare viene risolta grazie al cosiddetto effetto idrofobico. Questo spinge la parte proteica dell'enzima ad avvolgersi in modo tale da spingere i gruppi funzionali idrofili sulla superficie esterna a contatto con l'acqua e tutta la parte idrofoba all'interno, assumendo nel contempo quella struttura terziaria 
tenuta assieme da interazioni deboli (legami idrogeno, interazioni dipolo-dipolo, etc.) che è responsabile dell'attività catalitica dell'enzima e della straordinaria selettività di questi sistemi sia nei confronti del prodotto di reazione che del substrato da trasformare (Fig. 1A). La parte interna dell'enzima, dove di norma è collocato il sito attivo, costituisce quindi una nanofase idrofoba separata dall'acqua che fa da mezzo di reazione anche per reagenti idrofobi ( $\mathrm{R}$ in Fig. 1A).
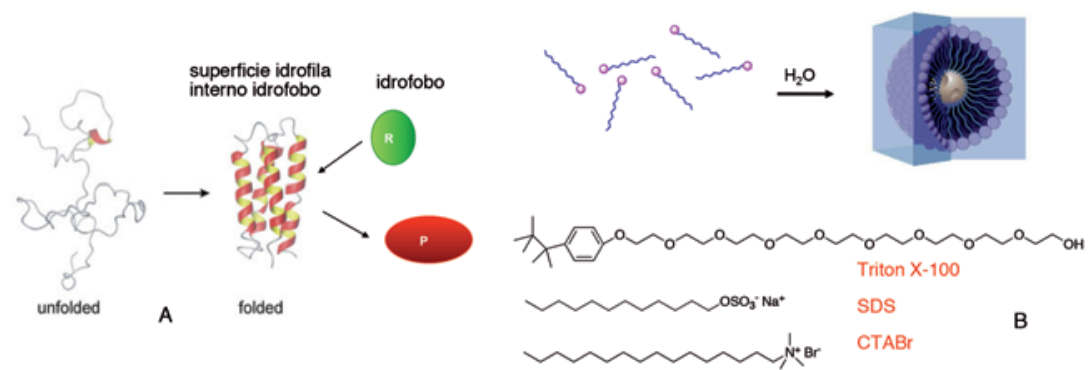

Fig. 1 - L'effetto idrofobico costringe l'enzima ad ad assumere la tipica struttura terziaria con $i$ gruppi idrofili rivolti verso l'esterno $(A)$ e le molecole di tensioattivo ad aggregarsi in micelle $(B)$.

La stessa strategia può essere mimata impiegando tensioattivi. I tensioattivi (la base dei comuni detergenti) sono molecole anfifiliche caratterizzate da una testa polare idrofila e una coda apolare idrofoba (Fig. 1B). Al di sopra di una certa concentrazione in acqua chiamata concentrazione micellare critica ( $\mathrm{cmc}$ ) esse si autoassemblano formando degli aggregati chiamati micelle in cui le teste idrofile sono poste sulla superficie a contatto con l'acqua, mentre le code si dispongono all'interno (Fig. 1B), comportandosi in questo come gli enzimi anche se con una complessità molecolare molto minore.

Le micelle possono essere viste come dei nanoreattori capaci di sciogliere catalizzatore e reagenti idrofobi già conosciuti per reazioni condotte in fase organica, senza la necessità di dover modificare i leganti presenti per rendere il sistema compatibile con l'acqua. Questi sistemi autoassemblati sono pertanto impiegabili in una gamma molto ampia di casi. La dissoluzione nelle micelle porta generalmente a concentrazioni locali maggiori, da cui dovrebbe conseguire un aumento della reattività del sistema, inoltre l'impiego a fine reazione di solventi organici immiscibili con acqua può consentire una facile estrazione dei 
prodotti di reazione ed aprire la strada alla possibilità di riciclare il catalizzatore con evidenti vantaggi economici e migliori prospettive di applicazione pratica.

\section{ALCUNI ESEMPI DI CATALISI IN SISTEMI MICELLARI}

Sulla base di queste considerazioni generali qualche anno fa abbiamo cominciato ad esplorare l'utilità dei sistemi acquosi micellari in una serie di reazioni catalitiche cominciando da alcune ossidazioni enantioselettive con acqua ossigenata impiegando catalizzatori chirali a base di platino. Si tratta di reazioni con un grande potenziale applicativo nella sintesi di farmaci e di prodotti naturali.[6] In Fig. 2 vengono riportati alcuni esempi di risultati ottenuti nella solfossidazione (A),[7] nell'ossidazione di Baeyer-Villiger di chetoni (B) [8] e nell'epossidazione di alcheni terminali (C).[9]

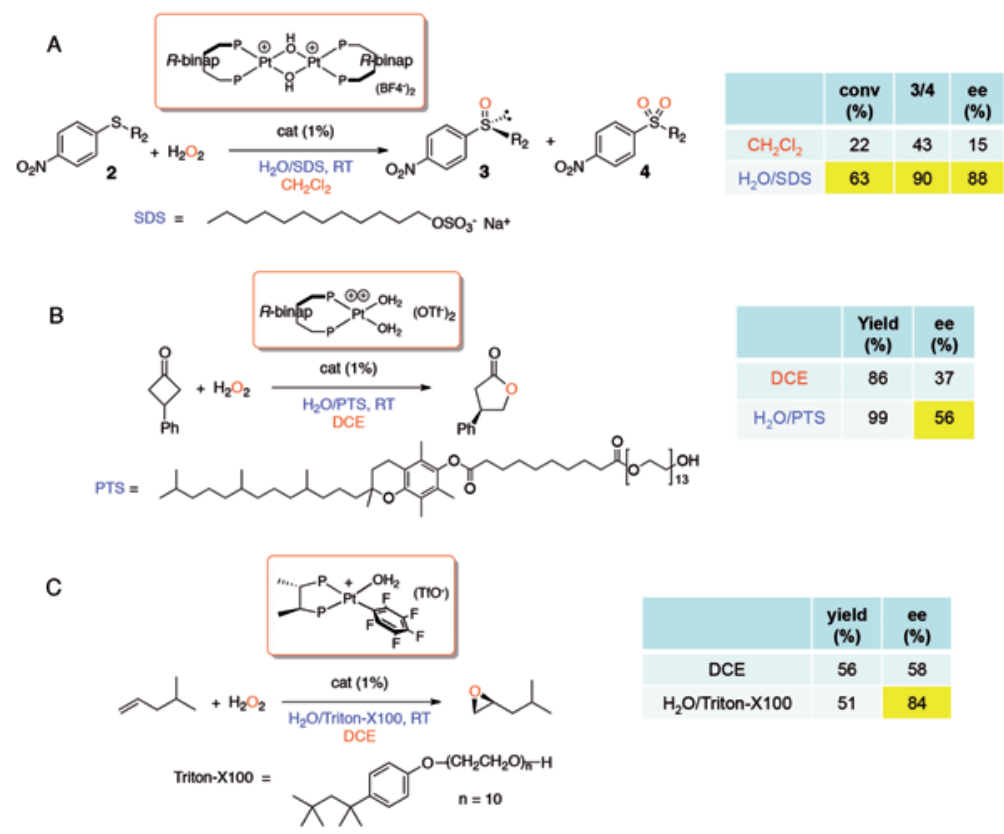

Fig. 2 - Ossidazioni enantioselettive con acqua ossigenata catalizzate da complessi cationici di platino. Confronto fra mezzi di reazione organici e micellari. 
A titolo di esempio viene mostrato il confronto fra il comportamento in mezzo organico e in mezzi micellari acquosi per alcuni substrati tipo. La scelta del tensioattivo più adatto è il risultato di un processo di ottimizzazione riguardante la natura e la concentrazione dello stesso che deve essere condotto per ogni specifico caso. Come si vede rispetto al mezzo organico l'uso del mezzo micellare porta sempre ad un miglioramento dell'enantioselettività della reazione ed in qualche caso (Fig. $2 A$ e, in misura minore, Fig. $2 B$ ) anche ad un miglioramento della resa in prodotto utile. Nel caso dell'epossidazione (Fig. 2C) è stato possibile separare il catalizzatore mediante estrazione con esano (immiscibile con acqua) e riciclarlo più volte senza osservare perdite di attività e di enantioselettività.

Spronati da questi risultati abbiamo provato le potenzialità del mezzo micellare nel caso di catalizzatori particolarmente inefficienti per verificare quanto poteva essere spinto il possibile miglioramento legato al nuovo mezzo di reazione. Qualche anno fa Katsuki,[10] studiando l'ossidazione di Baeyer-Villiger enantioselettiva di chetoni meso catalizzata da complessi di cobalto modificati con leganti di tipo salen (Fig. 3), aveva osservato che un requisito fondamentale per ottenere alte rese ed enantioselettività era la presenza di un anello a 7 termini comprendente gli atomi di azoto e il metallo e la presenza di gruppi elettron attrattori sugli anelli aromatici (catalizzatore di sinistra).
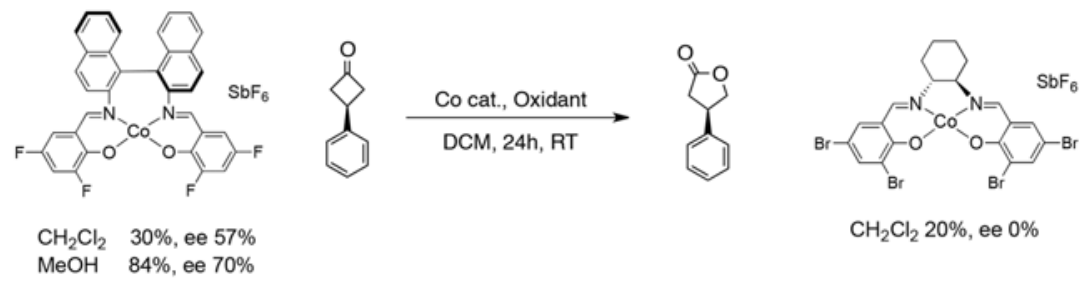

$\mathrm{CH}_{2} \mathrm{Cl}_{2} 20 \%$, ee $0 \%$

Fig. 3 - Ossidazione di chetoni catalizzata da complessi di Co(salen). Effetto dei sostituenti e della forma del legante.

Bastava un anello a 5 termini (catalizzatore di destra) per determinare un calo nella resa e l'annullamento dell'enantioselettività. Su questa base il catalizzatore di Co(salen) mostrato in Fig. 4, che contiene un anello a 5 termini e sostituenti elettron donatori, dovrebbe essere un vero 
disastro. In effetti, provato nell'ossidazione del chetone biciclico mostrato in Fig. 4, esso ha dimostrato una reattività scarsa e una capacità enantioselettiva pari a zero. Il semplice uso di un mezzo micellare $\mathrm{H}_{2} \mathrm{O}$ /Triton-X114 aumenta la resa in prodotto all' $80 \%$, migliora il rapporto fra i due diastereoisomeri $\mathbf{9}$ e $\mathbf{1 0}$ e l'enantioselettività passa da 0 all' $88 \%$.[11] Questo risultato particolarmente eclatante suggerisce che il nuovo mezzo micellare non è semplicemente un solvente diverso, ma introduce dei nuovi fattori in grado di controllare (spingere) il decorso della reazione catalitica.

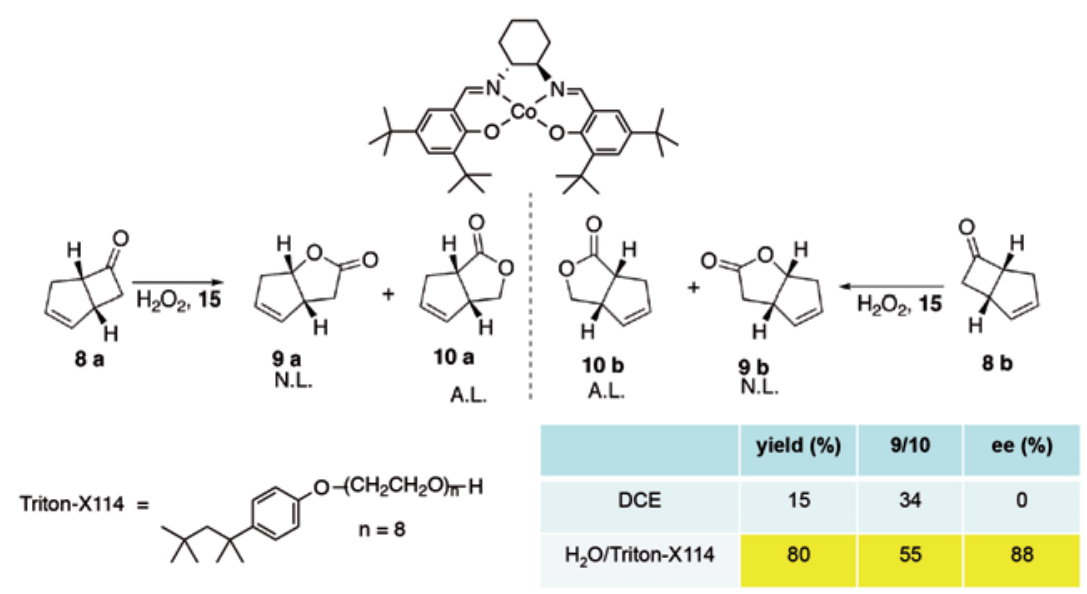

Fig. 4 - Ossidazione di chetoni catalizzata da complessi di Co(salen). Effetto del mezzo di reazione.

L'uso dei sistemi micellari non si è limitato alle reazioni di ossidazione, ma è stato esteso anche ad altre reazioni catalitiche non enantioselettive. A titolo di esempio in Fig. 5 vengono riportati due casi: uno di idratazione di nitrili con complessi di rutenio [12] e l'altro riguardante l'idroformilazione di alcheni terminali catalizzata da complessi di platino.[13] In entrambi i casi le rese sono molto alte e nettamente migliori rispetto alla stessa reazione condotta in fase organi$\mathrm{ca}$, il catalizzatore è riciclabile più volte senza perdita di attività e selettività, inoltre nel caso dell'idroformilazione la regioselettività aldeide lineare/aldeide ramificata è praticamente totale nei confronti dell'aldeide lineare. 

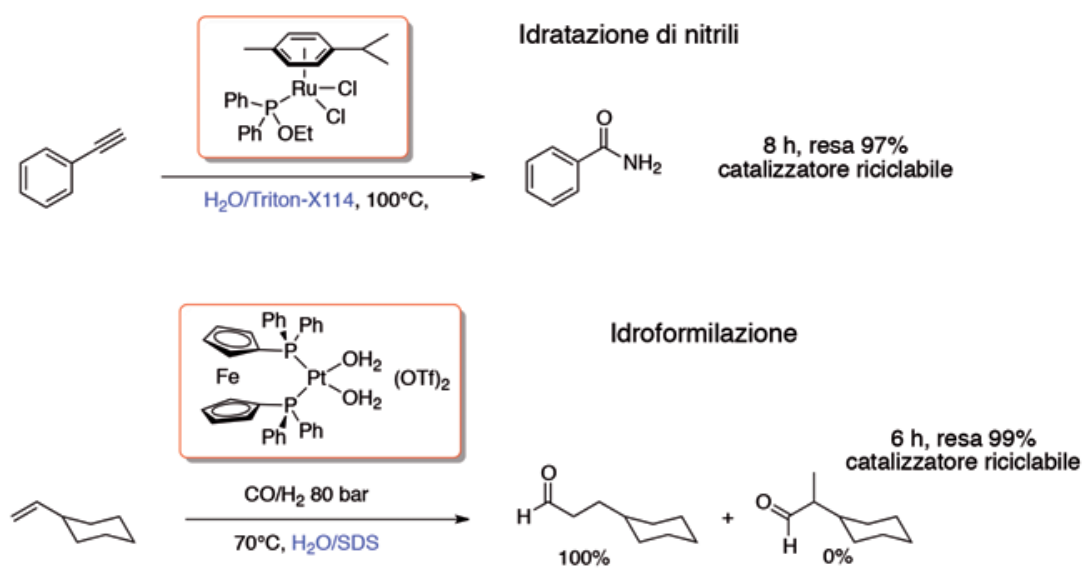

Fig. 5 - Idratazione di nitrili e idroformilazione di alcheni in mezzi micellari.

\section{Posizionamento del Catalizzatore}

Un aspetto importante in questi sistemi micellari per cercare di capire gli effetti osservati è il posizionamento del catalizzatore all'interno della micella. Per ottenere questo tipo di informazione di grande aiuto è l'uso di tecniche spettroscopiche NMR bidimensionali (NOESY e DOSY) che consentono di evidenziare le eventuali interazioni fra la molecola di catalizzatore e le varie porzioni della molecola di tensioattivo.

Le varie reazioni di ossidazione sopra riportate costituiscono un buon esempio per mettere in evidenza quanto questi sistemi siano sensibili al posizionamento del complesso che funge da catalizzatore. In tutti i casi sono stati impiegati catalizzatori di Pt(II) con caratteristiche molto simili: hanno tutti la stessa geometria di coordinazione, tutti contengono leganti fosfinici, tutti sono cationici, tutti sono insolubili in acqua e tutti vengono solubilizzati dall'aggiunta di tensioattivi. Tuttavia in presenza di micelle costituite da sodio dodecilsolfato (SDS) essi si posizionano in maniera molto diversa. Come hanno dimostrato gli studi NMR condotti su questi sistemi, i complessi [(P$\mathrm{P}) \mathrm{Pt}(-\mathrm{OH})]_{2}{ }^{2+}$ usati nella solfossidazione (Fig. $2 A$ ) interagiscono con il primo $\mathrm{CH}_{2}$ della catena alifatica adiacente alle teste anioniche del tensioattivo, stanno quindi sulla superficie esterna della micella;[7] i complessi $\left[(\mathrm{P}-\mathrm{P}) \mathrm{Pt}_{t}\left(\mathrm{C}_{6} \mathrm{~F}_{5}\right)\left(\mathrm{H}_{2} \mathrm{O}\right)\right]^{+}$usati nell'epossidazione di alcheni 
(Fig. 2C) stanno nel profondo della micella e interagiscono con i gruppi $\mathrm{CH}_{3}$ terminali della catena alifatica idrofoba; [9] infine i complessi $\left[(\mathrm{P}-\mathrm{P}) \mathrm{Pt}\left(\mathrm{H}_{2} \mathrm{O}\right)_{2}\right]^{2+}$ si collocano più o meno a metà strada fra la superficie e il centro e interagiscono con le porzioni $\mathrm{CH}_{2}$ della catena alifatica idrofoba.[8]

Queste differenze di comportamento hanno delle implicazioni che cercheremo di razionalizzare anche per fornire una base interpretativa agli effetti osservati sulla reattività dei catalizzatori.

\section{VANTAGGI DEI SISTEMI MICELLARI}

Quanto sin qui esposto si presta ad alcune considerazioni di carattere generale e, a questo punto, anche verificate sperimentalmente: (i) i mezzi micellari consento l'uso efficace dell'acqua come solvente per una varietà di reazioni catalitiche in fase omogenea; (ii) spesso consentono anche il riciclo del catalizzatore mediante estrazione con solventi organici e successiva separazione di fase; ovviamente il solvente scelto non deve sciogliere nè il tensioattivo nè il catalizzatore; i risultati migliori sinora sono stati osservati con esano; (iii) molto spesso i sistemi micellari aumentano l'attività e la selettività del catalizzatore rispetto a quanto già noto in ambiente organico; infine (iv) non esistono criteri validi che consentano di predeterminare la scelta del tensioattivo migliore e di prevedere il posizionamento del catalizzatore nella micella, ogni caso fa storia a sè e quindi la scelta del tensioattivo e la sua concentrazione ottimale vanno determinati di volta in volta.

La prima domanda che sorge spontanea è la seguente: come può essere spiegato l'aumento di attività catalitica? Una prima risposta deriva da un semplice calcolo considerando le quantità tipiche di catalizzatore, substrato, tensioattivo e solvente impiegate negli esperimenti. Si può facilmente dimostrare che le concentrazioni di catalizzatore e substrato in micella sono all'incirca di un ordine di grandezza maggiori rispetto all'uso del solvente organico. Quindi c'è un effetto di maggiore concentrazione che tende a spingere in su la velocità di reazione. Una seconda considerazione deriva dal fatto che all'interno della micella c'è una costante dielettrica diversa rispetto al solvente: questo implica un diverso effetto solvente che potrà essere favorevole o sfavorevole $\mathrm{a}$ seconda del tensioattivo scelto. La terza considerazione si basa sul fatto che lo stato di transizione della reazione potrebbe essere stabilizzato da 
un'interazione con i gruppi polari diminuendo l'energia di attivazione del processo e quindi aumentando la velocità di reazione.

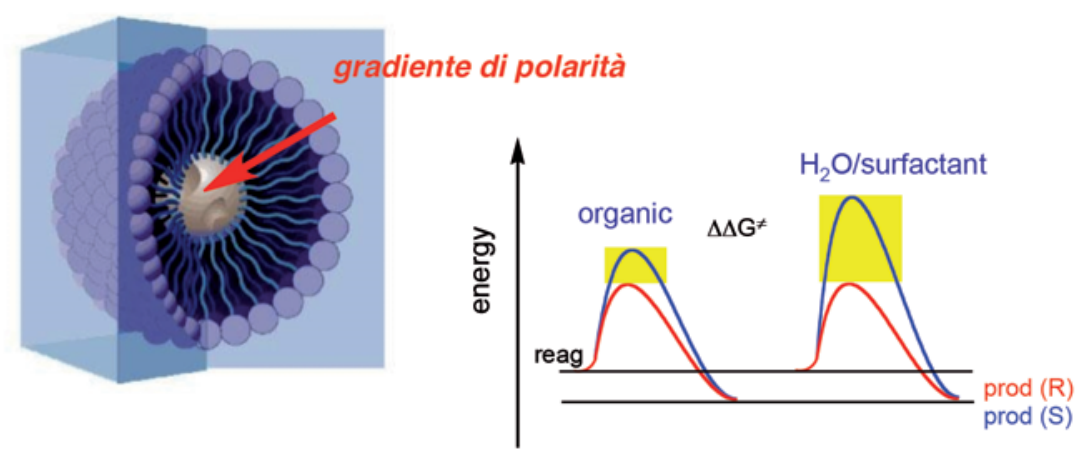

Fig. 6 - Gradiente di polarità all'interno della micella e sue conseguenze sulla stabilizzazione dello stato di transizione in reazioni enantioselettive.

La seconda domanda è: come si spiega l'aumento di enantioselettività osservato in molti casi? Se si considera la struttura di una micella appare chiaro che andando dalla superficie, dove sono presenti gruppi funzionali fortemente idrofili, verso il centro fortemente idrofobo si riscontra un gradiente di polarità (Fig. 6). Questo implica che catalizzatore e substrato saranno localizzati in posizioni diverse a seconda delle loro proprietà. La costrizione in uno spazio confinato ed anisotropo influenzerà la richiesta sterica ed elettronica dello stato di transizione della reazione e comporterà l'interazione con aree interne alla micella caratterizzate da diversa polarità. Questo può comportare un effetto differenziato sui due stati di transizione diastereoisomerici di una trasformazione enantioselettiva amplificando le differenze di energia di attivazione (Fig. 6). Queste considerazioni sono ovviamente estendibili a tutti i casi di selettività di prodotto (chemio-, regio-, stereo-, etc.). I dati sperimentali relativi all'eccesso enantiomerico osservato nelle varie reazioni qui riportate consentono di calcolare il valore di $\Delta \Delta G^{\neq}$fra i due stati di transizione che conducono all'enantiomero $\mathrm{R}$ e all'enantiomero $\mathrm{S}$. Ad es. il 58\% di ee osservato per l'epossidazione di 4-metil-2-pentene in dicloroetano riportato in Fig. $2 C$ equivale ad un $\Delta \Delta G^{\neq}$pari a $-3.23 \mathrm{~kJ} / \mathrm{mol}$, mentre l' $84 \%$ di ee osservato per la stessa reazione in acqua/SDS equivale ad un $\Delta \Delta G^{\neq}$pari a $-5.95 \mathrm{~kJ} / \mathrm{mol}$ con una differenza di circa 2.5 $\mathrm{kJ} / \mathrm{mol}$ nei due casi. Analogamente l'ossidazione di Baeyer-Villiger ripor- 
tata in Fig. 4 implica una differenza fra sistema micellare (88\% ee) e solvente organico $(0 \%$ ee) pari a $6.82 \mathrm{~kJ} / \mathrm{mol}$. Pertanto i sistemi micellari possono costituire un ausilio molto efficace nel promuovere reazioni (enantio)selettive.

\section{AfFINITÀ CON GLI ENZIMI}

Gli esempi qui riportati indicano come i sistemi micellari abbiano forti affinità con altri sistemi catalitici che producono i loro effetti all'interno di spazi confinati, come ad es. le zeoliti in cui la costrizione sterica può indurre effetti molto marcati nella distribuzione dei prodotti di una reazione. La shape selectivity non si esplica solo come capacità di selezionare i substrati all'ingresso dei pori della zeolite in base alle dimensioni e alla forma (setacci molecolari), ma anche nel controllo sterico all'interno delle gabbie nei confronti dello stato di transizione della reazione catalizzata.[14] Ad es. è stato visto che catalizzatori chirali ingabbiati all'interno di una zeolite o di un solido microporoso sono in grado di dispiegare una maggiore enantioselettività.[15] Tuttavia l'affinità maggiore dei sistemi micellari è con gli enzimi. Qui la selettività non è solo determinata da fattori sterici, ma anche dalle interazioni elettroniche che si instaurano con lo stato di transizione da parte dei gruppi funzionali che si trovano in prossimità del sito attivo. Diversamente dalle zeoliti, micelle ed enzimi non sono oggetti rigidi, quindi la presenza del catalizzatore in micella all'interno di uno spazio confinato ed anisotropo implica una grande "superficie di contatto" fra gli intermedi di reazione e l'aggregato circostante e questo, come si è visto, si può tradurre in una elevata reattività e selettività. Questo parallelismo può essere spinto oltre? può implicare ulteriori analogie in grado di indurre proprietà inusuali per i comuni catalizzatori omogenei?

Una della proprietà tipiche degli enzimi è la cosiddetta selettività di substrato. Gli enzimi oltre alla capacità di dirigere una reazione esclusivamente verso il prodotto desiderato (selettività di prodotto) hanno anche la capacità di selezionare, in mezzo ad una miriade di molecole organiche, anche omologhe, sciolte nel mezzo in cui operano (acqua), solo la molecola che deve essere trasformata (selettività di substrato) ed escludendo tutte le altre, sulla base di interazioni deboli di tipo sterico ed elettronico che portano il substrato giusto a percorrere il cammino che, partendo dalla superficie idrofila e attraverso la parte 
proteica dell'enzima, conduce sino al sito attivo, pre-orientano il substrato e lo preparano per la successiva trasformazione attraverso una serie di interazioni non covalenti che spaziano dai legami idrogeno alle forze di Van der Waals. E' il principio della chiave e della serratura suggerito da Emil Fischer nel 1894 (Fig. 7). I normali catalizzatori omogenei non godono di questa proprietà poichè sono circondati da un mezzo isotropo come il solvente e, in presenza di substrati con lo stesso gruppo funzionale da trasformare e con moderate differenze in parti remote della molecola, la loro capacità di selezione è praticamente trascurabile (Fig. 7).

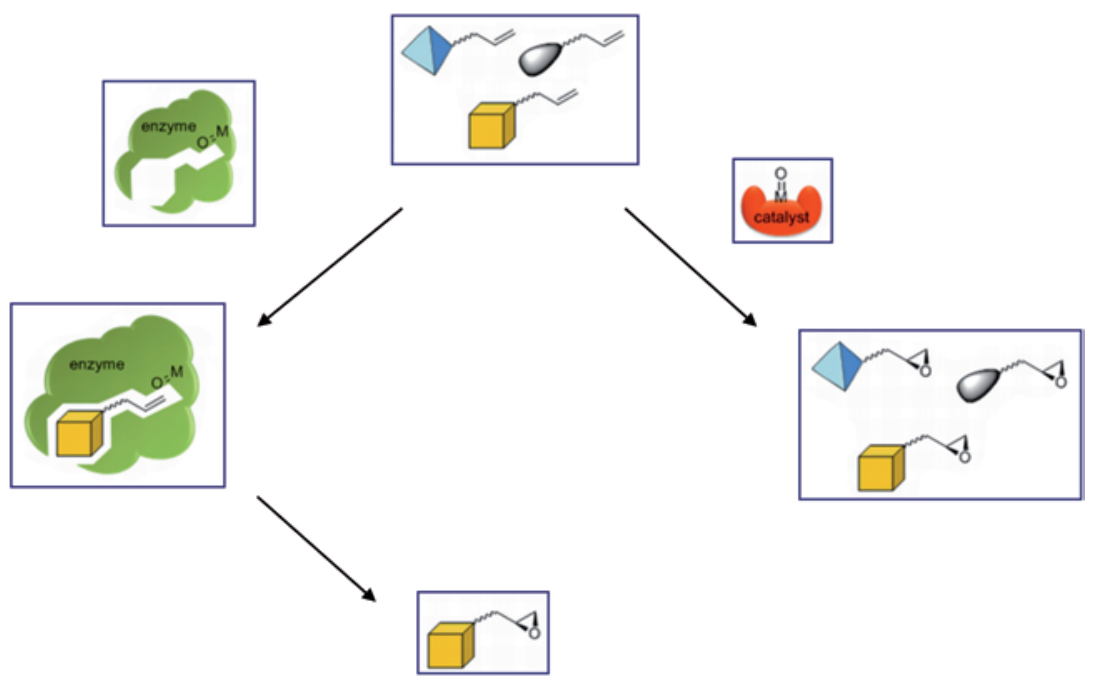

Fig. 7 - Selettività di substrato: comportamento degli enzimi e dei normali catalizzatori molecolari.

Per provare se questa capacità di indurre selettività di substrato era presente anche in sistemi acquosi micellari sono stati condotti degli esperimenti nella reazione di Diels-Alder fra ciclopentadiene e una serie di aldeidi $\alpha, \beta$-insature alifatiche in cui la catena viene progressivamente allungata da 1 fino a 7 atomi di carbonio (quindi aldeidi da $\mathrm{C}_{4}$ a $\mathrm{C}_{10}$ ) e tutte alimentate contemporaneamente nella stessa miscela di reazione per verificare la capacità discriminante del sistema catalitico (Fig. 8).[16] Il catalizzatore è un complesso di $\mathrm{Cr}$ (salen) (Fig. 8) sciolto o in cloroformio, oppure in un sistema micellare acqua/SDS. I risultati sono mostrati 
nel grafico di Fig. 8. Come si vede il complesso in ambiente organico è più attivo, ma la differenza di reattività fra l'aldeide $\mathrm{C}_{4}$ e l'aldeide $\mathrm{C}_{10}$ è praticamente trascurabile. Viceversa in ambiente micellare lo stesso catalizzatore risulta più lento, ma l'aldeide $\mathrm{C}_{10}$ risulta quattro volte più reattiva dell'aldeide $\mathrm{C}_{4}$. L'ambiente micellare idrofobo che circonda il catalizzatore seleziona preferenzialmente l'aldeide più lunga (più idrofoba) dal mezzo circostante e quindi rappresenta un primo esempio di selettività di substrato.

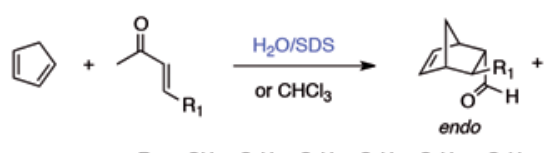

$\mathrm{R}_{1}=-\mathrm{CH}_{3},-\mathrm{C}_{2} \mathrm{H}_{5},-\mathrm{C}_{3} \mathrm{H}_{7},-\mathrm{C}_{4} \mathrm{H}_{9},-\mathrm{C}_{6} \mathrm{H}_{13},-\mathrm{C}_{7} \mathrm{H}_{15}$

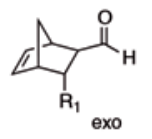

exo

$$
100
$$

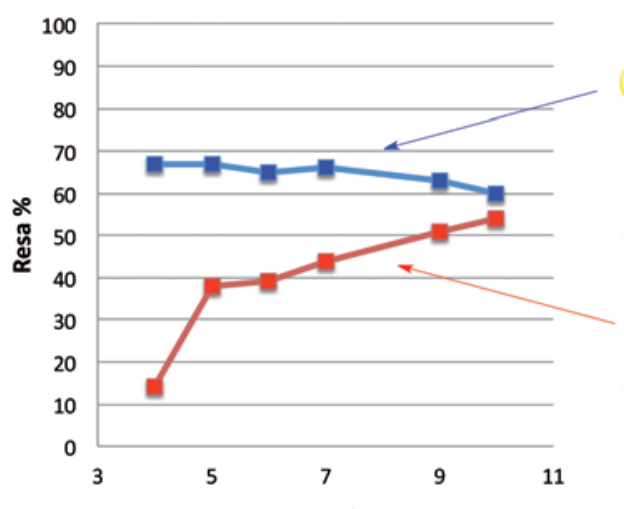

N. atomi C

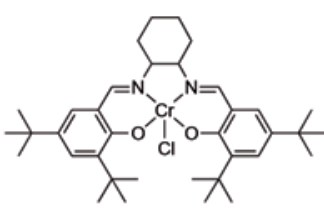

$(2 \% \mathrm{~mol})$
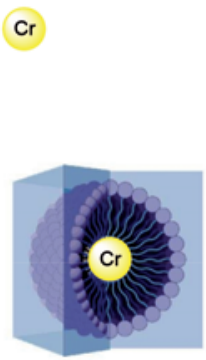

Fig. 8 - Reazione di Diels-Alder fra ciclopentadiene e una miscela di aldeidi $\alpha$, $\beta$-insature alifatiche catalizzata da un complesso di cromo. Confronto fra la reazione in fase organica e in fase micellare.

Lo stesso effetto è stato verificato anche nell'idrogenazione delle aldeidi $\alpha, \beta$-insature alifatiche sopra menzionate alle corrispondenti aldeidi sature, sempre alimentate come miscela, impiegando o tetraidrofurano (THF) come solvente organico oppure il sistema micellare acqua/SDS.[17] In questo caso è stato impiegato un catalizzatore di palladio colloidale ottenuto da $\mathrm{Pd}(\mathrm{OAc})_{2}$ posto sotto idrogeno che è risultato attivo anche $\mathrm{T}$ ambiente (Fig. 9). Il catalizzatore viene preven- 
tivamente preparato o in THF, oppure in $\mathrm{H}_{2} \mathrm{O}$ /SDS; in entrambi i casi si ottengono particelle di $\mathrm{Pd}$ di dimensioni nanometriche, più piccole e stabili nell'ambiente micellare. Quando la miscela di aldeidi è posta a contatto col catalizzatore sotto idrogeno si osservano gli effetti mostrati in Fig. 9. Come si vede quando il Pd viene impiegato in THF si osserva un calo di velocità di reazione di circa 3.5 volte passando dall'adeide $\mathrm{C}_{4}$ all'aldeide $\mathrm{C}_{10}$, viceversa quando lo stesso catalizzatore viene impegato in micella la velocità di reazione non solo segue un ordine inverso, ma l'aldeide $\mathrm{C}_{10}$ reagisce circa 340 volte più velocemente dell'aldeide $\mathrm{C}_{4}$. Ovviamente il mezzo di reazione diverso implica qualche differenza nei due casi, le principali sono: una maggiore solubilità dell'idrogeno in fase organica piuttosto che in acqua, la presenza di particelle di $\mathrm{Pd}$ presumibilmente più piccole in micella (diam. medio $5 \mathrm{~nm}$ ) piuttosto che in THF dove il Pd colloidale è poco stabile, ma soprattutto (come nel caso di Fig. 8) una maggiore affinità del mezzo micellare idrofobo per le aldeidi più lunghe, le cui proprietà idrofobe aumentano all'aumentare della lunghezza della catena. E' quest'ultimo il fattore determinante per spiegare la grande differenza di reattività osservata nei due casi.

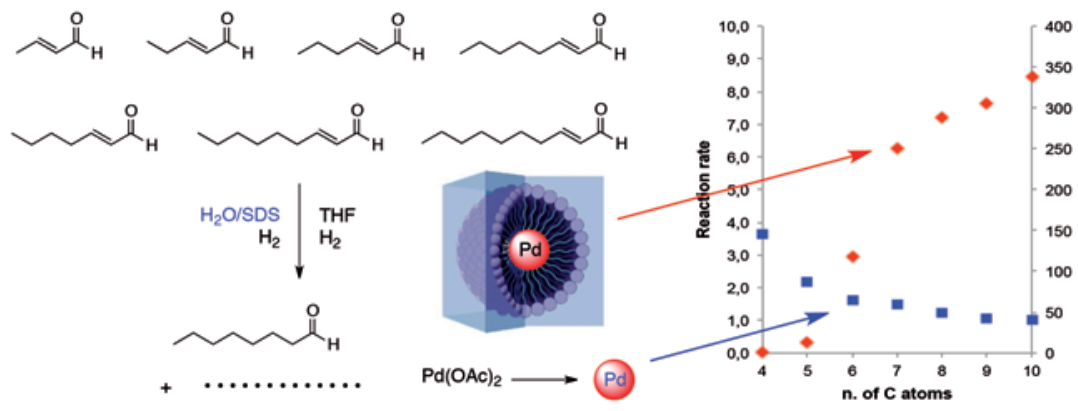

Fig. 9 - Idrogenazione di una miscela di aldeidi $\alpha$, $\beta$-insature alifatiche catalizzata da nanoparticelle di $\mathrm{Pd}$. Confronto fra la reazione in fase organica e in fase micellare.

\section{CONCLUSIONI}

In conclusione, in questa breve rassegna si è cercato di dimostrare come i sistemi micellari (essenzialmente acqua e sapone) possano essere un valido ausilio per la conduzione di reazioni catalitiche organiche e 
siano in grado di aprire la via all'uso dell'acqua come solvente per le stesse. Questo comporta una serie di conseguenze applicative molto importanti quali: la possibilità di eliminare i solventi organici con evidenti vantaggi in termini di economicità e protezione dell'ambiente, la possibilità di separare e riciclare il catalizzatore, prolungandone la vita e limitando il problema dell'inquinamento dei prodotti di reazione da parte dei metalli impiegati. In cascata questi due aspetti a loro volta implicano una semplificazione di processo legata al minor numero di operazioni di separazione e purificazione, minori costi legati all'uso del catalizzatore e alla necessità di smaltimento del solvente, in definitiva una maggiore sostenibilità sia in termini economici che ambientali.

Come si è visto le micelle sono sistemi che si autoassemblano nell'ambiente di reazione. I tensioattivi non richiedono nessuno sforzo sintetico particolare, sono semplicemente degli ingredienti che vengono aggiunti al sistema catalitico, dopodichè fanno tutto da soli. In genere sono poco costosi e la scelta è amplissima così come il loro uso attuale in settori quali la detergenza, la formulazione di cosmetici, di farmaci e nell'industria alimentare.

Concettualmente non ci sono limiti per il tipo di catalizzatore impiegato o la reazione scelta. In pratica ci sono ovviamente delle limitazioni: la più ovvia è che il catalizzatore impiegato non deve decomporsi o disattivarsi a contatto con l'acqua. Questo elimina una serie di catalizzatori a base di metalli fortemente ossofilici e tutte quelle reazioni che devono essere condotte in ambiente rigorosamente anidro. Per quanto riguarda le condizioni di reazione, le reazioni di idroformilazione qui presentate hanno dimostrato che pressioni fino a 80 bar e temperature fino a $100^{\circ} \mathrm{C}$ non determinano il collasso del sistema micellare, che anzi continua a funzionare senza problemi. Chiaramente per definire meglio questi limiti operativi questi sistemi dovranno essere applicati in una casistica più ampia.

La catalisi in questi sistemi confinati può offrire una serie di vantaggi rispetto all'uso di solventi organici tradizionali legati soprattutto alla maggiore attività catalitica e migliore selettività (chemio-, regio-, stereo-, enantio-, ...) dovute alla natura anisotropa del mezzo micellare che può stabilizzare lo stato di transizione della reazione, mimando in parte il comportamento degli enzimi.

Infine questo aspetto può indurre effetti sconosciuti nella catalisi tradizionale e caratteristici invece degli enzimi, come ad es. la selettività di substrato. Siamo quindi in presenza di una nuova metodologia cata- 
litica, che si aggiunge all'arsenale attualmente a disposizione del chimico organico di sintesi e le cui potenzialità sono ancora largamente inesplorate.

\section{BIBLIOGRAFIA}

[1] BM Trost Science 1991, 254, 1471.

[2] RA Sheldon Green Chem. 2007, 9, 1273.

[3] (a) C Seyler, C Capello, S Hellweg, C Bruder, D Bayne, A Huwiler, K Hungerbühler, Ind. Eng. Chem. Res. 2006, 45, 7700. (b) C Capello, U Fischer, K Hungerbühler, Green Chem., 2007, 9, 927.

[4] (a) UM Lindstrom, Chem. Rev. 2002, 102, 2751. (b) UM Lindstrom, Organic Reactions in Water, Blackwell, London, 2008.

[5] CW Kohlpainter, RW Fischer, B Cornils, Appl. Catal. A: General 2001, 221, 219.

[6] (a) A Scarso, G Strukul, Transition-metal-catalyzed stereoselective oxidations in drug and natural product synthesis, in "Stereoselective Synthesis of Drugs and Natural Products", V Andrushko and N Andrushko Eds, Wiley, London, 2013, Ch. 34; (b) A Scarso, G Strukul, Transition-metal-catalyzed asymmetric sulfoxidation in drug and natural product synthesis, in "Stereoselective Synthesis of Drugs and Natural Products", V Andrushko and N Andrushko Eds, Wiley, London, 2013, Ch. 48.

[7] A Scarso, G Strukul, Adv. Synth. Catal., 2005, 347, 1227-1234.

[8] A Cavarzan, G Bianchini, P Sgarbossa, L Lefort, S Gladiali, A Scarso, G Strukul, Chem. Eur. J., 2009, 15, 7930-7939.

[9] (a) M Colladon, A Scarso, P Sgarbossa, RA Michelin, G Strukul, J. Am. Chem. Soc., 2006, 128, 14006-14007. (b) M Colladon, A Scarso, G Strukul, Adv. Synth. Catal. 2007, 349, 797-801.

[10] T Katsuki, T Uchida Tetrahedron Lett. 2001, 42, 6911-6914.

[11] G Bianchini, A Cavarzan, A Scarso, G Strukul, Green Chem., 2009, 11, 1517.

[12] A Cavarzan, A Scarso, G Strukul, Green Chem., 2010, 12, 790-794.

[13] M Gottardo, A Scarso, S Paganelli, G Strukul, Adv. Synth. Catal., 2010, 352, 2251-2262.

[14] J Hagen, Industrial Catalysis: A Practical Approach, Wiley-VCH, Weinheim, 1999, ch 6, pp 230-235.

[15] (a) C Li, Catal. Rev., 2004, 46, 419-492. (b) JM Thomas, T Maschmeyer, BFG Johnson, DS Shephard, J. Mol. Catal. A: Chem., 1999, 141, 139-144; (c) MD Jones, R Raja, JM Thomas, BFG Johnson, Top. Catal., 2003, 25, 71-79; (d) H Zhang, Y Zhang, C Li, J. Catal., 2006, 238, 369-381.

[16] F Trentin, A Scarso, G Strukul, Tetrahedron Lett., 2011, 52, 6978-6981.

[17] G LaSorella, P Canton, A Scarso, G Strukul, Chem. Commun., submitted. 